\title{
Development of Quadrupole Mass Spectrometers Using Rapid Prototyping Technology
}

\author{
Boris Brkić, ${ }^{a}$ Neil France, ${ }^{a}$ Adam T. Clare, ${ }^{\mathrm{b}}$ Chris J. Sutcliffe, ${ }^{\mathrm{b}}$ \\ Paul R. Chalker, ${ }^{b}$ and Stephen Taylor ${ }^{\mathrm{a}}$ \\ a Department of Electrical Engineering and Electronics, University of Liverpool, Liverpool, United Kingdom \\ ${ }^{b}$ Department of Engineering, University of Liverpool, Liverpool, United Kingdom
}

In this report, we present a prototype design of a quadrupole mass filter (QMF) with hyperbolic electrodes, fabricated at the University of Liverpool using digital light processing (DLP), a low-cost and lightweight 3D rapid prototyping (RP) technique. Experimental mass spectra are shown for $\mathrm{H}_{2}{ }^{+}, \mathrm{D}_{2}{ }^{+}$, and $\mathrm{He}^{+}$ions to provide proof of principle that the DLP mass filter is working as a mass analyzer in the low-mass range (1 to $10 \mathrm{amu}$ ). The performance of the DLP QMF has also been investigated for individual spectral peaks. Numerical simulations of the instrument were performed by coupling CPO and Liverpool QMS-2 programs to model both the ion source and mass filter, respectively, and the instrument is shown to perform as predicted by theory. DLP thus allows miniaturization of mass spectrometers at low cost, using hyperbolic (or other) geometries of mass analyzer electrodes that provide optimal ion manipulation and resolution for a given application. The potential of using RP fabrication techniques for developing miniature and microscale mass analyzers is also discussed. (J Am Soc Mass Spectrom 2009, 20, 1359-1365) (c) 2009 American Society for Mass Spectrometry

$\mathrm{D}$ evelopment of mass spectrometers has advanced rapidly in recent years with a focus on building fully integrated devices that can be made portable while maintaining good performance. To achieve this, miniaturization of mass spectrometer components has been pursued, especially in the case of mass analyzers. This is because reduction in size of analyzers offers several advantages:

1. Lower manufacturing costs are possible because the implementation technologies used often offer mass production not only for separate components, but also for complete devices.

2. Operation at higher pressures because of shorter length of the ion mean free path.

3. Use of smaller and less expensive vacuum systems attributed to smaller device size and higher pressure operation.

4. Lower power consumption with the possibility of operation using low-power battery systems since lower electrode voltages are needed to generate the required electric fields.

5. Potential for the whole mass spectrometry system to be made portable.

Address reprint requests to Dr. Boris Brkić, University of Liverpool, Department of Electrical Engineering and Electronics, Brownlow Hill, Liverpool, Merseyside L69 3GJ, United Kingdom. E-mail: Boris.Brkic@ liv.ac.uk
Therefore, these advantages will further increase the number of mass spectrometry applications, particularly for field applications such as instant medical diagnosis, water and environmental analysis, and detection of oil, natural gas, and explosives [1].

In the past, miniaturization of mass spectrometers had been done by using several different methods. First of all, there are semiconventional methods, which were used for realization of miniature quadrupole mass filters (QMFs) and arrays of quadrupole analyzers for residual gas analysis [2-5]. Another approach includes miniaturization of hyperbolic QMFs using ceramic material, coated with metal to define electrode regions [6, 7]. In recent years, the most popular method for miniaturization of mass analyzers is microelectromechanical systems (MEMS) technology, which is mainly based on semiconductor microfabrication processes. Since complex electrode geometries such as hyperbolic are difficult to fabricate at the microscale using MEMS, simpler geometries such as cylindrical and planar are used to give approximation to ideal hyperbolic field. Miniature mass analyzers constructed using MEMS include QMFs with cylindrical electrodes [8-10], time-of-flight mass analyzers [11], and several types of ion traps [12-15]. Ion source MEMS miniaturization has also advanced with realization of a carbon nanotube electron impact ion source [16]. Finally, some rapid prototyping techniques have also been used for miniaturization of mass analyzers, including an ultraviolet-LIGA process for 
fabricating two-dimensional (2D) QMF arrays [17] and stereolithography (SLA) for fabricating rectilinear ion traps [18]. An attractive goal for most of the previously mentioned miniaturization methods is a reliable and fully integrated mass spectrometer with all of its components built using the same process.

Here, we propose a novel approach for miniaturization of quadrupole mass spectrometers (QMS) using rapid prototyping techniques. The technique used for fabrication of a hyperbolic QMF is digital light processing (DLP), which was initially invented by Texas Instruments (TI, Dallas, TX, USA) for video applications. DLP is based on a TI digital micromirror device (DMD), which is a MEMS semiconductor chip that contains microscopic mirrors aligned on a matrix. The purpose of micro mirrors is to enable precise control of the laser beams to achieve high-resolution projection stereolithography for fabricating complex three-dimensional (3D) microstructures $[19,20]$. DLP uses materials such as polymethyl methacrylate (PMMA) to produce any 3D shape with low fabrication errors at microscale. We have used DLP to fabricate PMMA hyperbolic electrodes for a QMF and housing for them. QMF electrodes were then coated with gold to provide electrical conductivity with low surface roughness for both the PMMA rods and the gold coating. The reason we chose to fabricate a QMF with hyperbolic rods is that it can provide significantly better resolution at $10 \%$ of the peak height than the QMF with circular rods. This had been demonstrated experimentally by Brubaker [21], with an improvement of a factor of two in resolution, as well as numerically by Gibson [22], who quantified improvement in both resolution and transmission when hyperbolic electrodes are used compared with circular electrodes.

The following sections describe the methods used for DLP QMF fabrication, obtaining proof of principle for mass spectrometry operation and its performance investigation. Discussion follows of the advantages of using DLP for low-cost manufacturing not only for single mass analyzers with improved electromagnetic fields, but also for other segmented structures such as ion sources, prefiltered mass analyzers, and arrays of analyzers (e.g., trap arrays). Another advantage of DLP - the potential for a fully integrated and lightweight miniature mass spectrometer-is also discussed.

\section{Experimental}

\section{Modeling}

Both analytical and numerical modeling of electrostatic fields are useful and often essential when designing ion sources and mass analyzers because they simulate the results that appear in real systems. Our numerical model may be used to produce individual mass peaks or complete mass spectrum and it can support any type of ion source together with QMF with electrodes of any profile. The model works by coupling the commercially available CPO3D program [23] with an in-house simulation program suite the QMS-2 [24].

$\mathrm{CPO}$ is an electrostatic simulation package based on the boundary-element method (BEM), which has proved to be more accurate than the finite-element method (FEM) and the finite-difference method (FDM) for modeling electrostatic lenses [25]. It has also been shown that CPO is more accurate than SIMION (FDM) for modeling miniature ion traps in free space [26]. This is mainly because the BEM uses only the surface of the electrodes to define the grid points for calculating potentials, whereas the FEM and the FDM also use the space enclosed by the electrodes. In this way, the BEM enables faster computation and higher accuracy even with a small number of electrode segments, which are used to adjust the simulation accuracy. Another advantage is that the number of segments can be defined for different electrode regions and thus have larger numbers of segments for most critical regions and smaller numbers of segments for regions where high accuracy is not needed.

QMS-2 is a 2D simulation package for QMF, developed by the University of Liverpool and was initially based on the FDM and, more recently, including the BEM for calculating electric fields and potentials. It supports QMFs with hyperbolic, cylindrical, and square electrodes, giving accurate performance predictions [22, $24,27]$, including the effect of electrode misalignment [28]. The purpose of the QMS-2 is to generate individual mass peaks and full mass spectra for given ion masses within a specified mass range for different stability zones. This is done by defining QMF dimensions and drive parameters (voltages and frequency) as well as initial ion oscillation parameters (positions, energies, and velocity components). The initial ion parameters in QMS-2 can be defined by setting constant ion energy angular spread in the direction at which ions enter the QMF. Alternatively, a custom approach can be used by modeling ion motion within a given ion source using programs such as CPO and SIMION to obtain entrance ion parameters. This has a more accurate correspondence to a real system, especially for ion energies that are directly dependent on the voltages applied to the ion source lenses.

For this work, CPO was used to simulate ion trajectories for the electron impact ion source (EIIS) and QMS-2 was used for the hyperbolic QMF that was fabricated using rapid prototyping DLP. A hyperbolic QMF simulation was also added to CPO simulation of the EIIS to include the effect of the fringing fields as ions enter the mass filter. The effect of space charge was also simulated between ions within the ion source.

\section{Design}

Before fabrication of the hyperbolic QMF, a detailed computer-aided design (CAD) drawing was done in Pro/ENGINEER [29] to define the dimensions for every component. Such CAD drawings are directly loaded 
into the DLP system (described in the following text), which then manufactures desired 3D shapes according to the Pro/ENGINEER design. Figure $1 \mathrm{a}$ and $\mathrm{b}$ show the design drawing for the assembly of the hyperbolic QMF including electrodes, electrode housing, and pins. The shape of the grooves on the housing was carefully chosen to ensure tight fitting for the rods to establish a good alignment and uniform separation. This is especially important for QMFs where even a small displacement of the electrodes along the $y$-axis can severely reduce the performance of the instrument [28]. The pins were designed to enable electrical connection to the electrodes through the tiny holes and to further secure the electrodes within the housing.

\section{Fabrication Technique}

As mentioned previously, fabrication of the hyperbolic QMF was done using a DLP lithographic technique, based on the DMD. DLP has a dynamic masking capability to selectively expose photosensitive, resinous materials such as PMMA, causing them to polymerize layer by layer to realize a desired geometry. Unlike conventional lithographic techniques that require a specific mask per given pattern layer, at a significant cost in

(a)

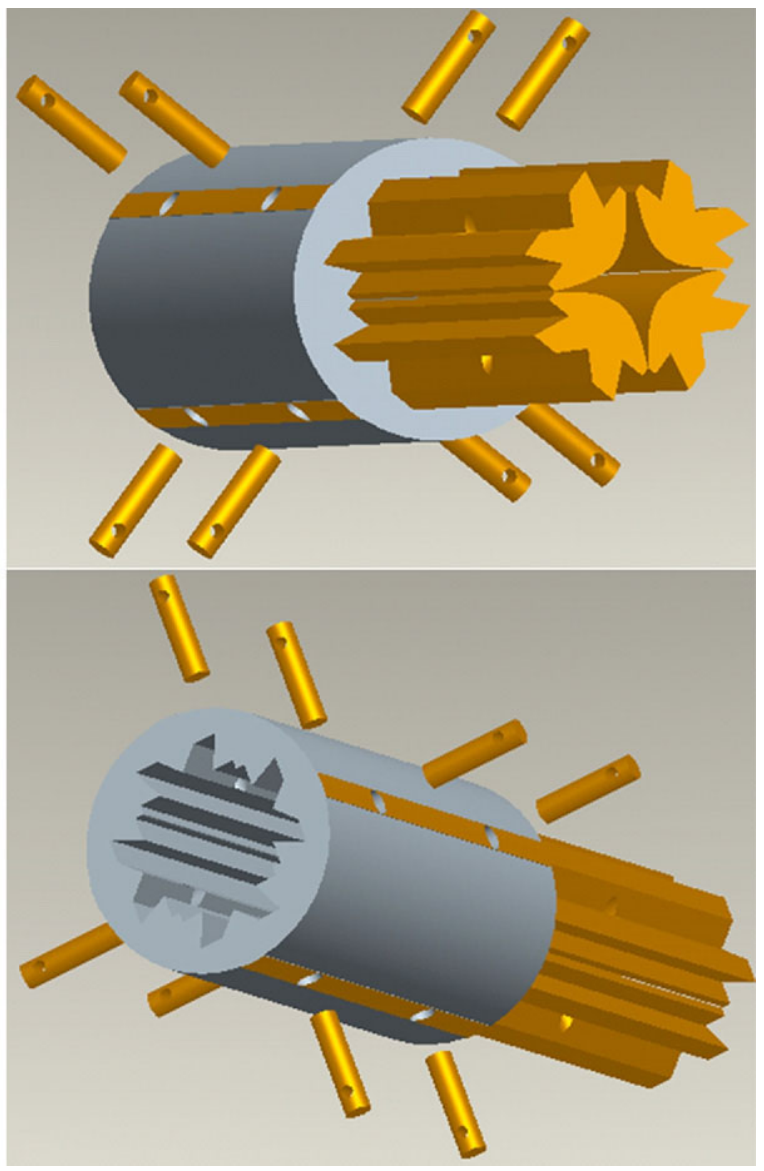

Figure 1. Pro/ENGINEER CAD design drawings for the assembly of the hyperbolic DLP QMF: showing QMF electrode design (a) and design for electrode grooves on the housing (b). most cases, with the DLP technique masking for each given layer is simply reprogrammed. In this way, the DLP uses a dynamic masking regime to induce repeated layers of a controlled geometry layer by layer.

Variations on the DLP theme are commonplace within the rapid prototyping (RP) market, although most systems consist of the following: DMD chassis (plus optics), stepper motor (for z-direction translations), resin container (with transparent floor), and a removable built substrate. The preprocessing of the computer-generated model for DLP is identical to that of the generic RP model, which makes it well suitable for mass production. The resolution of DLP systems is anisotropic. This is because resolution-limiting parameters are different in the coordinate axis with reference to each built layer. Geometries realized in this way can be thought of as consisting of a multitude of voxels (volumetric pixels). These discrete "building blocks" have their dimensions forced on them by the dimensions of each mirror mounted on the DMD (after focusing as observed at the resin floor), corresponding to the $x-y$ resolution and in the $z$-direction the smallest possible translation of the stepper motor. For our DLP structures, the $x-y$ voxel resolution used was $20 \mu \mathrm{m}$ and in the $z$-direction, $25 \mu \mathrm{m}$.

Good tolerances/surface finish, minimal secondary processing, and quick component interassembly are all advantages of DLP. The relative juvenility of DLP as a manufacturing technique means that its full potential is not yet realized and that considerable research needs to be taken within various institutions and companies. For our DLP QMF, a commercial envisionTEC Perfactory System (Gladbeck, Germany) was used to fabricate pins, electrodes, and the housing for them. Gold coating for the PMMA pins and electrodes was done by thermal evaporation with an Edwards E306A Coating System (Wilmington, MA, USA).

\section{Experimental Setup}

The fully assembled DLP QMF was coupled to a custom-built electronic control unit (ECU) and tested in a miniature vacuum system. The ECU was used to control the ion source voltages, to provide radiofrequency (rf) and dc drive voltages for the mass filter and to measure the current in the Faraday cup detector. It was linked via USB to a laptop PC for display of the mass spectra obtained. The vacuum system consisted of an Edwards two-stage rotary pump and an Edwards turbomolecular pump (for pressures down to $5 \times 10^{-6}$ Torr) with total pressure monitored using a vacuum gauge (Leybold Ionivac $\mathrm{GmbH}$, Cologne, Germany).

\section{Results and Discussion}

The hyperbolic QMF, fabricated using DLP, was designed to fit into our existing vacuum flange and metal housing as well as with our existing EIIS and detector. 
The DLP QMF has $r_{0}=2 \mathrm{~mm}$, where $r_{0}$ is half of the shortest distance between the opposing electrodes of the QMF. Length of the electrodes tested was $50 \mathrm{~mm}$. A commercial EIIS, built with conventional engineering, was used to test the DLP QMF. The EIIS consists of a cage where ions are generated, an extracting lens attached to the cage to release ions, a focusing lens to focus ions toward the QMF, and a decelerating lens held at ground to reduce ion energies before they enter the QMF. The cage has a cylindrical shape with diameter of $6 \mathrm{~mm}$ and length of $10 \mathrm{~mm}$. All three lenses have $0.3-\mathrm{mm}$ thickness and $0.8-\mathrm{mm}$ separation between them, where the decelerating lens is separated from the QMF by $0.5 \mathrm{~mm}$. All the lenses have $r_{e}=1.5 \mathrm{~mm}$, where $r_{e}$ is the exit aperture radius.

Figure 2a shows uncoated and coated DLP QMF rods after fabrication. The thickness of the gold coating was initially about $1 \mu \mathrm{m}$, which proved to be sufficiently high to allow QMF operation. The resistance of the conducting electrodes from one end to another in initial designs was roughly $40 \Omega$. By increasing the thickness of the gold coating, the resistance along the electrodes was reduced to only $0.1 \Omega$, which gave more

(a)

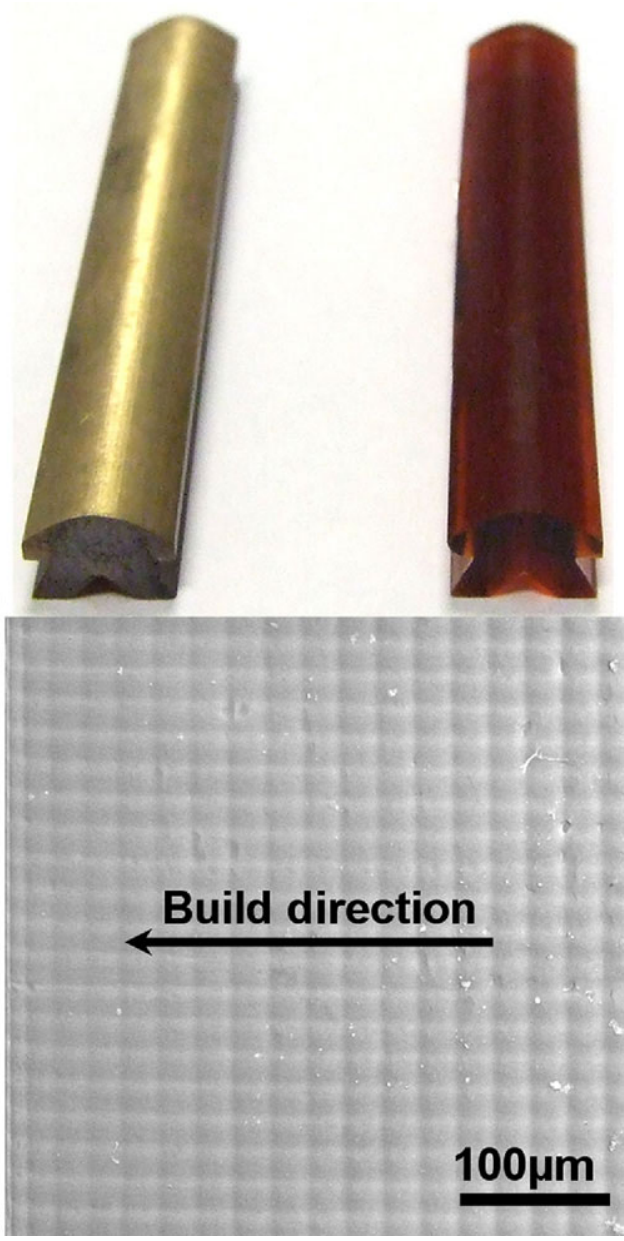

Figure 2. Gold-coated and non-coated electrodes of the DLP QMF (a) and microscopic image of the non-coated PMMA rod to show voxels and build direction (b). accurate driving voltages and enabled better optimization of spectral peaks. We did notice that after continuous use, the gold coating can wear slightly from the PMMA. To overcome this problem in the future, we propose to coat the electrodes with titanium, and then with gold, which should provide a better interface with PMMA. Figure $2 b$ shows a picture of the enlarged part of the noncoated PMMA rod, taken by scanning electron microscope. The surface roughness of the DLP rods was close to $1.5 \mu \mathrm{m}$, measured using a Wyko NT 3300 profiling system (white light interferometry; Veeco Instruments, Inc., Plainview, NY, USA). The roughness of electrode surfaces is especially important for miniature mass analyzers, where patch potentials can increase ion motional heating if electrodes are not sufficiently smooth, thus causing the distortion of ion motion [30].

Figure 3a shows the cross section of the assembly of the DLP QMF with $r_{0}=2 \mathrm{~mm}$. The unmetallized PMMA material of the housing behaves as a good insulator. Grooves within the housing were accurately fabricated so that the precision of electrode alignment is relatively high with around $1 \%$ error. Figure $3 \mathrm{~b}$ shows the enlarged image of the cross section of the hyperbolic DLP QMF, confirming good electrode alignment at the ends.

For initial QMS testing and for showing proof of principle, the QMF was driven at a frequency of 3.686 MHz. Ion source cage and the extracting lens were held at $3 \mathrm{~V}$, the focusing lens was held at $-39 \mathrm{~V}$, and the decelerating lens was at $0 \mathrm{~V}$. The emission current for ionization was $0.8 \mathrm{~mA}$ and operating pressure was $1.2 \times 10^{-4}$ Torr. Figure 4a shows an experimental mass spectrum for a $\mathrm{H}_{2} / \mathrm{D}_{2} / \mathrm{He}$ equal-concentration gas mixture measured using a commercial QMS with circular electrodes having prefilter and postfilters, manufactured by MKS Instruments (MKS Cirrus, Andover, MA, USA). The MKS QMS has $r_{0}=3.175$, electrode length of $100 \mathrm{~mm}$, and is driven at $1.843 \mathrm{MHz}$. The MKS QMS is tuned to about 1 -amu resolution at $10 \%$ of the peak height across the operating mass range. The resolution for $\mathrm{H}_{2}{ }^{+}$ions at mass 2 is 3.17 and the resolution for $\mathrm{D}_{2}{ }^{+}$ and $\mathrm{He}^{+}$ions, which are not resolved at mass 4 , is 5.88 at $10 \%$ of the peak height. Figure $4 \mathrm{~b}$ shows a complete experimental mass spectrum for the same $\mathrm{H}_{2} / \mathrm{D}_{2} / \mathrm{He}$ gas mixture obtained from the DLP QMS. The resolution for $\mathrm{H}_{2}{ }^{+}$ions at mass 2 is 3.03 and the resolution for $\mathrm{D}_{2}{ }^{+}$and $\mathrm{He}^{+}$ions at mass 4 is 5.13 at $10 \%$ of the peak height. A very good correlation between the ratios of spectral peaks can be seen by comparing the mass spectra from the commercial MKS QMS with the DLP QMS. This provides proof of principle of the DLP QMS instrument in the technologically important 1 to 10 amu mass range. The ECU used was originally designed for microscale mass filters (submillimeter $r_{0}$ ) and provides only low-output voltages and reduced mass range. By reducing the size of $r_{0}$ in future instrument realization, the mass range of the DLP instrument can be extended using the existing ECU. 
(a)

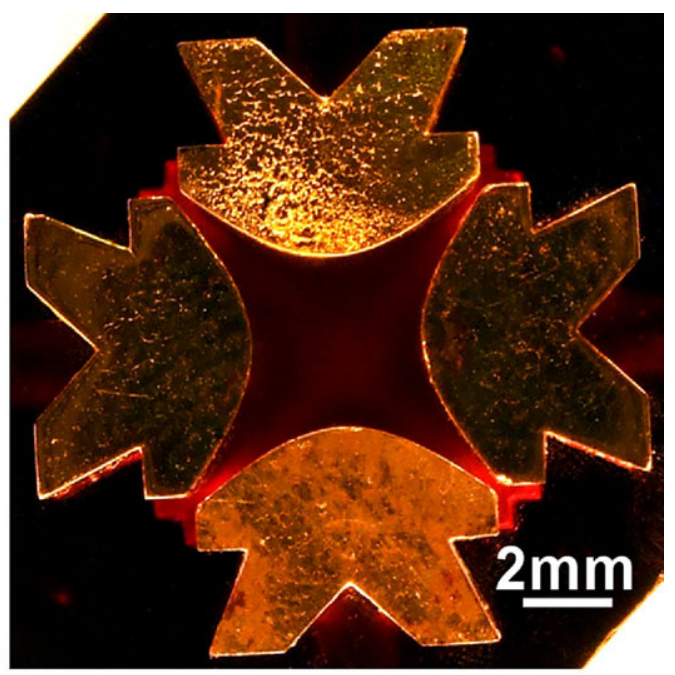

(b)

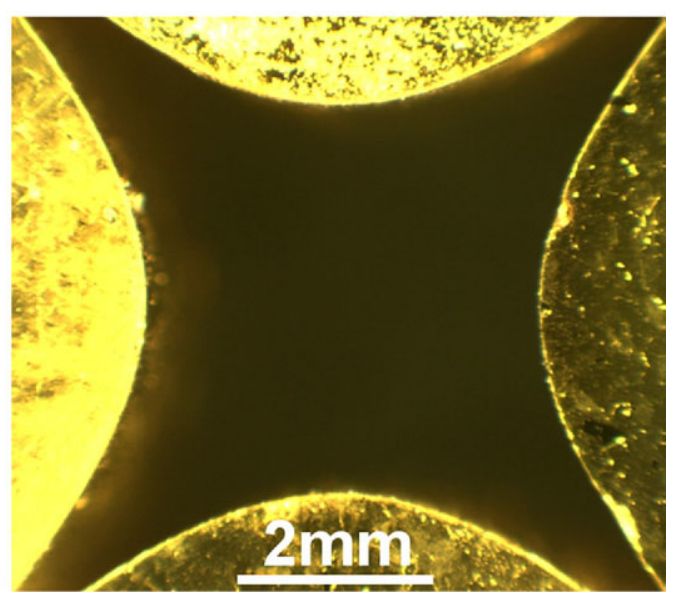

Figure 3. Cross sections of the DLP QMF prototype: showing the assembly (a) and electrode alignment (b).

To assess performance of the DLP QMF, optimizations of spectral peaks at mass 2 and 4, respectively, were obtained by altering the extracting voltage on the ion source cage (ion energy) and by changing the $U / V$ ratio. For optimization of a mass 2 peak, the cage voltage was varied in five steps with values of 4, 3, 2.5, 2 , and $1.5 \mathrm{~V}$, where the lowest voltage provides the lowest ion energy and highest resolution. The mass analyzer was driven at $3.686 \mathrm{MHz}$. Figure 5a shows the optimal simulated mass peak for ${ }^{2} \mathrm{H}^{+}$ions within the hyperbolic DLP QMF operating in zone 1 stability region with $1.5 \mathrm{~V}$ on the cage, obtained using $\mathrm{CPO}$ and QMS-2 programs. The simulated instrument resolution at $10 \%$ of the peak height is 33.33 . Figure $5 \mathrm{~b}$ shows the corresponding experimental mass peak for ${ }^{2} \mathrm{H}^{+}$ions with resolution of 30.5 at $10 \%$ of the peak height, which is almost as high as the theoretical prediction. Comparing Figure 5a and b, a close similarity can be seen between the shapes of the simulated and experimental mass peaks for $\mathrm{m} / \mathrm{z}=2$ as well as low-ion transmission ascribed to low ion energies. Figure 6a shows the optimization steps for increased resolution mass 2 peaks obtained by reducing the ion source cage extraction voltage. Figure $6 \mathrm{~b}$ shows variation of ion peak current with instrument resolution for mass ${ }^{2} \mathrm{H}^{+}$ ions for the given optimization steps. Figure $6 \mathrm{c}$ shows variation of ion peak current with instrument resolution for $m / z=4$ by varying $U / V$ ratio in five steps with values of $0.158,0.160,0.162,0.164$, and 0.166 . The cage extraction voltage was set to $1.2 \mathrm{~V}$ and the mass analyzer was driven at $2 \mathrm{MHz}$ to obtain mass 4 peaks. The highest resolution of 69.45 at $10 \%$ of the peak height was achieved with the highest $U / V$ ratio that corresponds to the peak of the zone 1 stability diagram, resulting in the lowest ion peak current.

We have also investigated outgassing of the PMMA material and noticed the increase from our system base pressure of $5 \times 10^{-6}$ Torr with an empty vacuum system to $2 \times 10^{-5}$ Torr with the DLP QMF inside, but we did not see any negative effects to the measured mass spectra and performance of the instrument in this mass range. Even though the DLP QMF was positioned near the electron impact ion source, we did not see any damage to the electrodes and assembly housing.

Apart from the ability to reproduce low-cost miniature structures with good tolerances and smooth surfaces, another major advantage of the DLP over other similar technologies is that it can be used for building segmented structures. For example, ion source lenses can easily be made with DLP, where the lens and the interlens insulator can be built as one polymer part, making a significant simplification. In this way, by
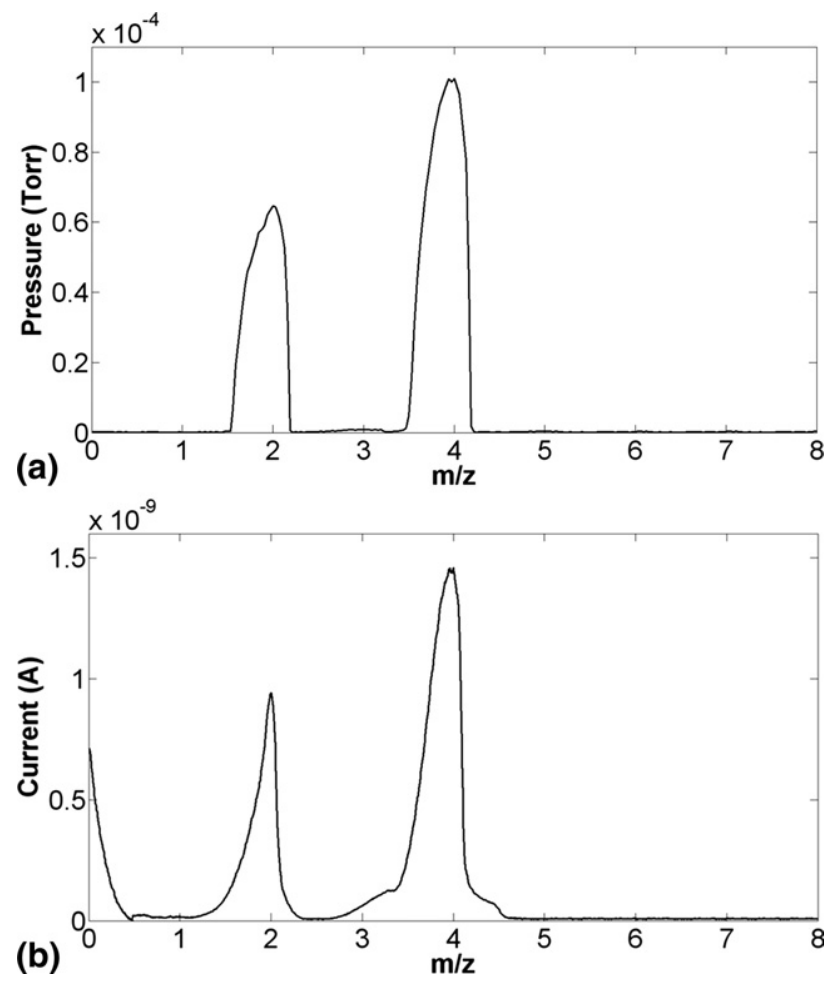

Figure 4. Experimental mass spectra for $\mathrm{H}_{2} / \mathrm{D}_{2} / \mathrm{He}$ gas mixture: showing spectrum obtained from the commercial MKS QMS with circular electrodes (a) and spectrum obtained from the hyperbolic DLP QMF (b). 

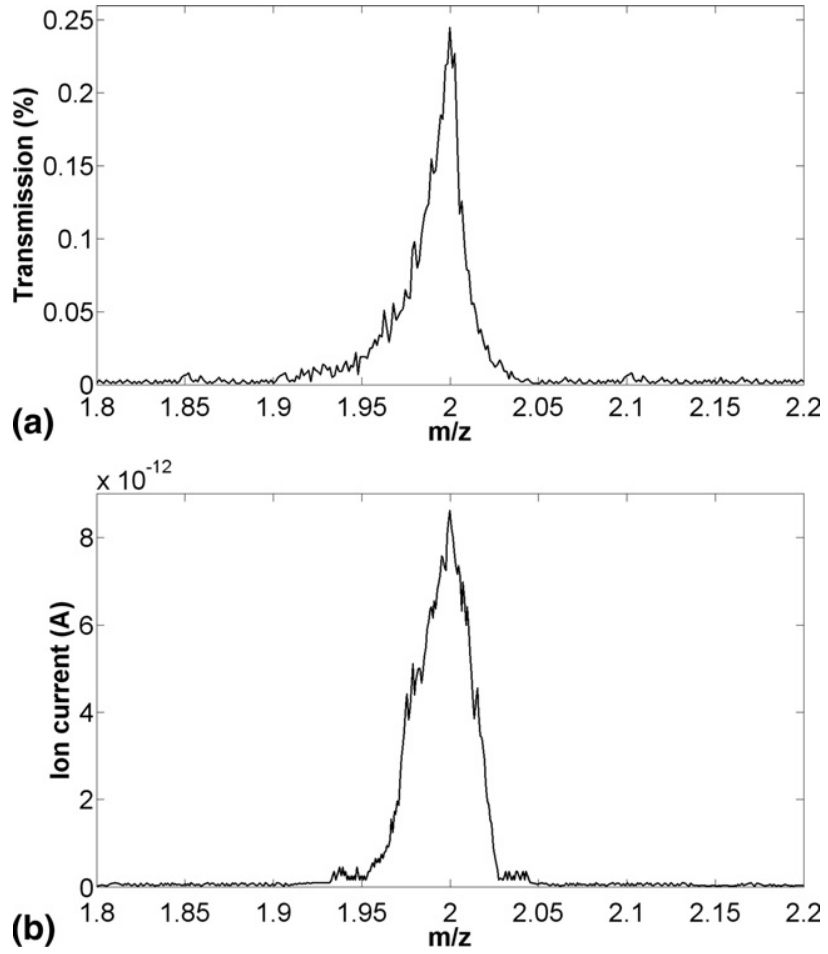

Figure 5. Mass peaks for ${ }^{2} \mathrm{H}^{+}$ions for the hyperbolic DLP QMF: showing optimal simulated peak, generated by coupling $\mathrm{CPO}$ and Liverpool QMS-2 programs (a) and optimal experimental peak, obtained by reduction of the ion source extraction voltage (b).

using a suitable mask, only segments that need to be conductive can be coated with gold (e.g., exit hole), while leaving other parts insulated. Another example of using DLP for segmented systems is for quadrupole mass filters that use prefilters for better sensitivity and postfilters for better detection. In this case, the electrodes of prefilters, main analyzer, and postfilters would all be part of the same PMMA rod, which would be coated accordingly to define conducting regions for each of these devices. In the same way, linear ion traps or trap arrays can be implemented in DLP so that rf and dc electrodes are all part of the same polymer structure. DLP structures with multiple parts will be significantly lighter than their conventional counterparts made with metal and ceramic insulators. This is advantageous for applications where light weight is important, such as spacecraft.

\section{Conclusions}

A hyperbolic quadrupole mass filter has been fabricated using a digital light processing technique and assembled with a conventional electron impact ion source and Faraday detector into a quadrupole mass spectrometer (QMS). Experimentally obtained mass spectra for a $\mathrm{H}_{2} / \mathrm{D}_{2} / \mathrm{He}$ gas mixture was obtained and found to agree both quantitatively and qualitatively with spectra obtained using a commercial triple-filter QMS for the same gas mixture, proving the principle of DLP QMS operation. QMS performance was obtained as expected from full simulation of the instrument, including ion source effects using a combination of commercial and in-house simulation software.

The DLP technique was found to be particularly suitable for implementing mass analyzers that require ideal hyperbolic fields or for any other devices with complex geometries. Compared to other rapid prototyping techniques, such as selective laser melting and selective laser ablation, DLP offers significantly smaller feature size that allows a higher degree of accuracy. It also has the potential to produce fully integrated mass spectrometers with a single mass analyzer or with an array of analyzers. Future work will focus on realization of mass analyzers and other mass spectrometer components at the submillimeter and micro scales.
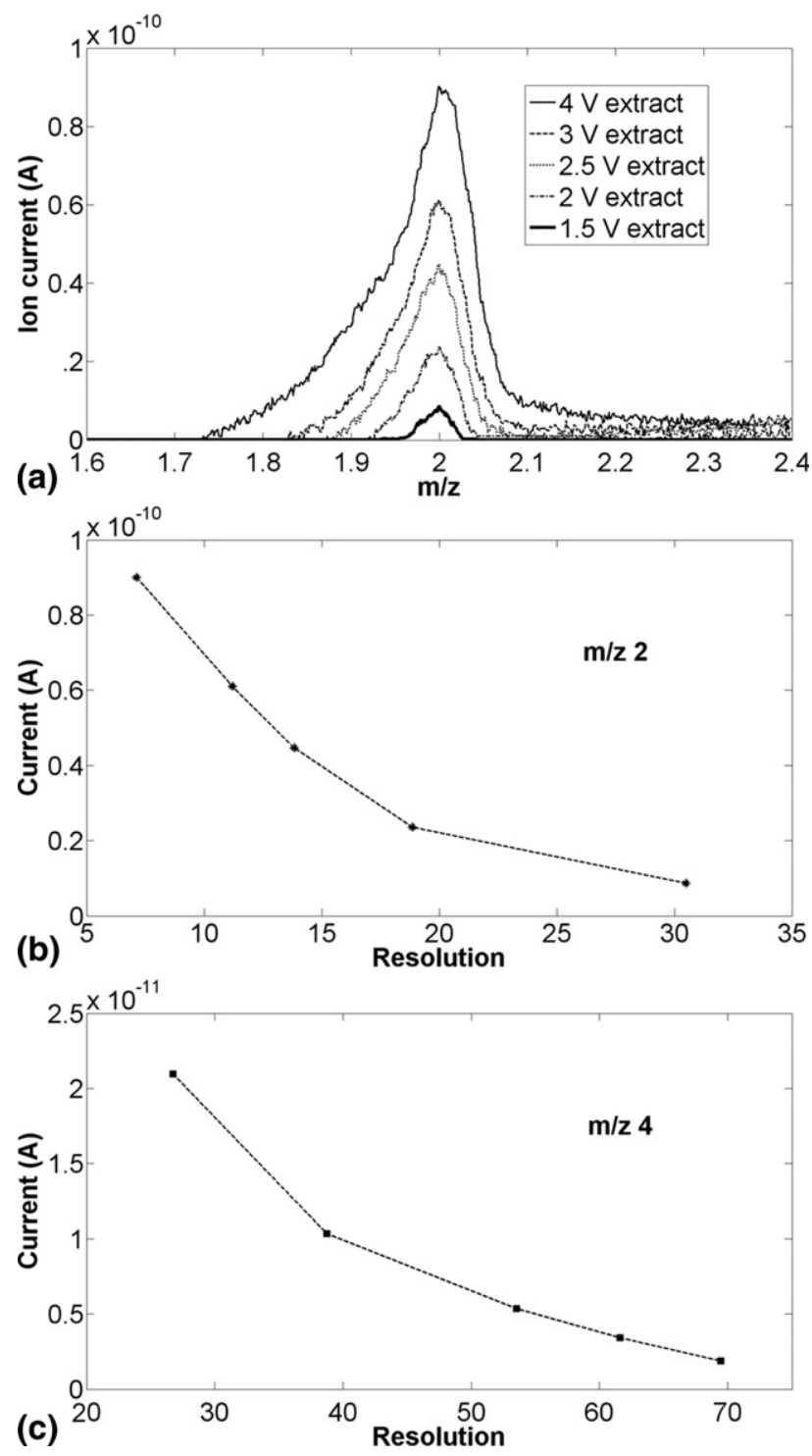

Figure 6. Experimental optimizations of the hyperbolic DLP QMF spectral peaks: optimization of mass 2 peaks by altering the ion source cage extraction voltage (a), and experimental variation of ion peak current with instrument resolution for mass 2 (b) and for mass 4 (c). 


\section{Acknowledgments}

We gratefully acknowledge EPSRC (UK) for funding this work under the Follow on Fund programme.

\section{References}

1. Taylor, S.; Bierbaum, V. M. Focus on Harsh Environment Mass Spectrometry. J. Am. Soc. Mass Spectrom. 2008, 19, 1375-1376.

2. Ferran, R. J.; Boumsellek, S. High-Pressure Effects in Miniature Arrays of Quadrupole Analyzers for Residual Gas Analysis from $10^{-9}$ to $10^{-2}$ Torr. J. Vac. Sci. Technol. A 1996, 14, 1258-1265.

3. Holkeboer, D. H.; Karandy, T. L.; Currier, F. C.; Frees, L. C.; Ellefson, R. E. Miniature Quadrupole Residual Gas Analyzer for Process Monitoring at milliTorr Pressures. J. Vac. Sci. Technol. A 1998, 16, 1157-1162.

4. Boumsellek, S.; Ferran, R. J. Miniature Quadrupole Arrays for Residual and Process Gas Analysis. I. Inst. Environ. Sci. Technol. 1999, 42, 27-31.

5. Ketola, R. A.; Kiuru, J. T.; Tarkiainen, V.; Kotiaho, T.; Sysoev, A. A. Comparison of Analytical Performances of a Micro-Array Quadrupole Instrument and a Conventional Quadrupole Mass Spectrometer Equipped with Membrane Inlets. Rapid Commun. Mass Spectrom. 2003, $17,753-756$

6. Hiroki, S.; Abe, T.; Murakami, Y.; Takano, Y.; Higuchi, M.; Miyake, M. Development of a QMS with a Ceramic Single-Piece Quadrupole. Vacuum 1993, 44, 71-74.

7. Wang, J.; Zhang, X.; Mao, F.; Xiao, M.; Cui, Y.; den Engelsen, D.; Lei, W. Study of a Micro Chamber Quadrupole Mass Spectrometer. J. Vac. Sci. Technol. A 2008, 26, 239-243.

8. Taylor, S.; Tindall, R. F.; Syms, R. R. A. Silicon-Based Quadrupole Mass Spectrometry Using Microelectromechanical Systems. J. Vac. Sci. Technol. B 2001, 19, 557-562.

9. Geear, M.; Syms, R. R. A.; Wright, S.; Holmes, A. S. Monolithic MEMS Quadrupole Mass Spectrometers by Deep Silicon Etching. IEEE/ASME J. Microelectromech. Syst. 2005, 14, 1156-1166.

10. Velasquez-Garcia, L. F.; Akinwande, A. I. An Out-of-Plane MEMS Quadrupole for a Portable Mass Spectrometer. Proc. Transducers Conf. 2007, 2315-2320.

11. Wapelhorst, E.; Hauschild, J. P.; Müller, J. Complex MEMS: A Fully Integrated TOF Micro Mass Spectrometer. Sens. Actuators A 2007, 138, 22-27.

12. Blain, M. G.; Riter, L. S.; Cruz, D.; Austin, D. E.; Wu, G.; Plass, W. R.; Cooks, R. G. Towards the Hand-Held Mass Spectrometer: Design Considerations, Simulation, and Fabrication of Micrometer-Scaled Cylindrical Ion Traps. Int. J. Mass Spectrom. 2004, 236, 91-104.

13. Pau, S.; Pai, C. S.; Low, Y. L.; Moxom, J.; Reilly, P. T. A.; Whitten, W. B.; Ramsey, J. M. Microfabricated Quadrupole Ion Trap for Mass Spectrometer Applications. Phys. Rev. Lett. 2006, 96, 120801.
14. Van Amerom, F. H. W.; Chaudhary, A.; Cardenas, M.; Bumgarner, J.; Short, R. T. Microfabrication of Cylindrical Ion Trap Mass Spectrometer Arrays for Handheld Chemical Analyzers. Chem. Eng. Comm. 2008, 195, 98-114.

15. Austin, D. E.; Wang, M.; Tolley, S. E.; Maas, J. D.; Hawkins, A. R.; Rockwood, A. L.; Tolley, H. D.; Lee, E. D.; Lee. M. L. Halo Ion Trap Mass Spectrometer. Anal. Chem. 2007, 79, 2927-2932.

16. Bower, C. A.; Gilchrist, K. H.; Piascik, J. R.; Stoner, B. R.; Natarajan, S.; Parker, C. B.; Wolter, S. D.; Glass, J. T. On-Chip Electron Impact Ion Source Using Carbon Nanotube Field Emitters. Appl. Phys. Lett. 2007, 90, 124102 .

17. Wiberg, D. V.; Myung, N. V.; Eyre, B.; Shcheglov, K.; Orient, O. J.; Moore, E.; Munz, P. LIGA-Fabricated Two-Dimensional Quadrupole Array and Scroll Pump for Miniature Gas Chromatograph/Mass Spectrometer. Proc. SPIE 2003, 4878, 8-13.

18. Fico, M.; Yu, M.; Ouyang, Z.; Cooks, R. G.; Chappell, W. J. Miniaturization and Geometry Optimization of a Polymer-Based Rectilinear Ion Trap. Anal. Chem. 2007, 79, 8076-8082.

19. Takahashi, T.; Akahane, T.; High Speed Rapid Prototyping using a Digital Micromirror Device. Tech. Digest 17th Sensor Symposium 2000, 271-274.

20. Sun, C.; Fang, N.; Wu, D. M.; Zhang, X. Projection Micro-Stereolithography Using Digital Micro-Mirror Dynamic Mask. Sens. Actuators A 2004, 121, $113-120$.

21. Brubaker, W. M. NASA Report. NASW 1970, 1298

22. Gibson, J. R.; Taylor, S. Prediction of Quadrupole Mass Filter Performance for Hyperbolic and Circular Cross Section Electrodes. Rapid Commun. Mass Spectrom. 2000, 14, 1669-1673.

23. CPO programs, available at www.electronoptics.com.

24. Hogan, T. J.; Taylor, S. Performance Simulation of a Quadrupole Mass Filter Operating in the First and Third Stability Zones. IEEE Trans. Instr. Meas. 2008, 57, 498-508.

25. Cubric, D.; Lencova, B.; Read, F. H.; Zlamal, J. Comparison of FDM, FEM and BEM for Electrostatic Charged Particle Optics. Nucl. Instr. Methods Phys. Res. A 1999, 427, 357-362.

26. Brkić, B.; Taylor, S.; Ralph, J. F.; France, N. High-Fidelity Simulations of Ion Trajectories in Miniature Ion Traps Using the Boundary-Element Method. Phys. Rev. A 2006, 73, 012326.

27. Gibson, J. R.; Taylor, S. Asymmetrical Features of Mass Spectral Peaks Produced by Quadrupole Mass Filters. Rapid Commun. Mass Spectrom. 2003, 17, 1051-1055.

28. Taylor, S; Gibson, J. R. Prediction of the Effects of Imperfect Construction of a QMS Filter. J. Mass Spectrom. 2008, 43, 609-616.

29. Pro/ENGINEER program, available at www.ptc.com.

30. Turchette, Q. A.; Kielpinski, D.; King, B. E.; Leibfried, D.; Meekhof, D. M.; Myatt, C. J.; Rowe, M. A.; Sackett, C. A.; Wood, C. S.; Itano, W. M.; Monroe, C.; Wineland, D. J. Heating of Trapped Ions from the Quantum Ground State. Phys. Rev. A 2000, 61, 063418. 